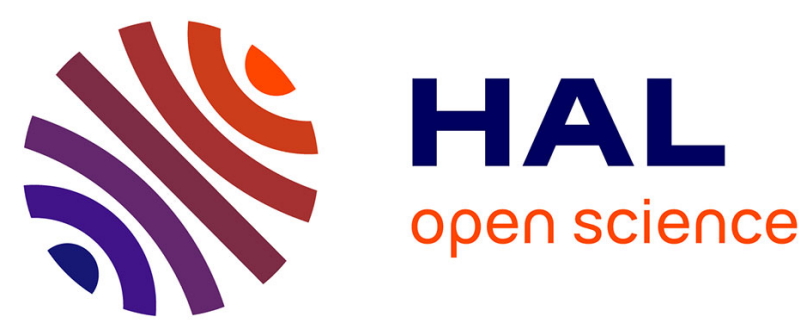

\title{
Light Memory Operation Based on a Double Pin SiC Device
}

\author{
V. Silva, M. Barata, M. A. Vieira, P. Louro, M. Vieira
}

\section{To cite this version:}

V. Silva, M. Barata, M. A. Vieira, P. Louro, M. Vieira. Light Memory Operation Based on a Double Pin SiC Device. 6th Doctoral Conference on Computing, Electrical and Industrial Systems (DoCEIS), Apr 2015, Costa de Caparica, Portugal. pp.265-272, 10.1007/978-3-319-16766-4_29 . hal-01343493

\section{HAL Id: hal-01343493 \\ https://inria.hal.science/hal-01343493}

Submitted on 8 Jul 2016

HAL is a multi-disciplinary open access archive for the deposit and dissemination of scientific research documents, whether they are published or not. The documents may come from teaching and research institutions in France or abroad, or from public or private research centers.
L'archive ouverte pluridisciplinaire HAL, est destinée au dépôt et à la diffusion de documents scientifiques de niveau recherche, publiés ou non, émanant des établissements d'enseignement et de recherche français ou étrangers, des laboratoires publics ou privés. 


\title{
Light Memory Operation Based on a Double Pin SiC Device
}

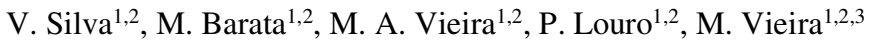 \\ ${ }^{1}$ Electronics Telecommunication and Computer Dept. ISEL, Lisboa, Portugal \\ ${ }^{2}$ CTS-UNINOVA, Quinta da Torre, Monte da Caparica, 2829-516, Caparica, Portugal \\ ${ }^{3}$ DEE-FCT-UNL, Quinta da Torre, Monte da Caparica, 2829-516, Caparica, Portugal
}

\begin{abstract}
Experimental optoelectronic characterization of a p-i'(a-SiC:H)-n/p$\mathrm{i}(\mathrm{a}-\mathrm{Si}: \mathrm{H})-\mathrm{n}$ heterostructure with low conductivity doped layers shows the feasibility of tailoring channel bandwidth and wavelength by optical bias through back and front side illumination. Front background enhances light-todark sensitivity of the long and medium wavelength range, and strongly quenches the others. Back violet background enhances the magnitude in short wavelength range and reduces the others. Experiments have three distinct programmed time slots: control, hibernation and data. Throughout the control time slot steady light wavelengths illuminate either or both sides of the device, followed by the hibernation without any background illumination. The third time slot allows a programmable sequence of different wavelengths with an impulse frequency of $6000 \mathrm{H}_{\mathrm{z}}$ to shine upon the sensor. Results show that the control time slot illumination has an influence on the data time slot which is used as a volatile memory with the set, reset logical functions.
\end{abstract}

Keywords: Optoelectronics, volatile memory, set reset logical functions.

\section{Introduction}

A volatile memory is a state variable that only maintains its value over a period of time and then its value changes without any intervention on the system. A decisive change of the state variable, at any time, will behave with the same manner. The time during which the state variable value is recoverable and the number of times it can be read without changing its value define the efficiency of the memory. Different memory technologies can be combined to produce innovative memories like one that uses light to read the sate variable [1], or molecules [2].

The aim of this document is to answer the research question: how is it possible to build a memory using a pi'inpin device?

The hypothesis, based on previous research of the characteristics of the pi'inpin device [3], points out that charges are stored by illuminating the device in the front or back surfaces, and by using this characteristic it is possible to build a volatile memory. Experimental results have shown that different light sequences within a set of fixed tuples produce different outputs for the same tuples. This points out that there is a direct influence of the previous tuple upon the current one. Understanding this behavior will determine if the pi'npin presents itself as an effective volatile memory. 


\section{Contribution to Cloud-based Engineering Systems}

The cloud, an icon used to represent the Internet, is now a service provided by many enterprises that host data space and offer applications that virtualizes storage and data bases. The Cloud is used in many circumstances has a common spot where to different users share data. It is a valuable asset for research as a means of connecting and sharing experimental data among researchers, so that validation can be performed, or to distribute tasks. Cloud services are "services delivered over the network" [4].

Another appealing usage is the possibility that experimental setup can be linked into the Cloud, so that researchers use the same setup thus reducing costs and accessing instruments and devices that need skills which they do not necessarily have or need in their research. This would provide an out-of-the-lab research, especially to inaccessible areas or with hazard settings forbidden for researchers.

The experiments and device presented in this paper fits into that scenario; building an application that is hosted in the Cloud would connect an authorized researcher to our laboratory. The laboratory would have the necessary instruments switched on and controlled by a computer connected to the Cloud. The researcher would then set the inputs and expect the experimental results. Every experiment would the stored into a database in the Cloud where any researcher could see past experiments, comment results and suggest improvements.

\section{Device Design, Characterization and Operation}

The device consists of a $1 \mathrm{~cm}^{2}$ double pin/pin a-SiC:H photo detector with front and back optical bias shown in

Fig. 1. The device operates within the visible range and input channels of several wavelengths (R: 626nm), green (G: $524 \mathrm{~nm})$, blue (B: $470 \mathrm{~nm})$ and violet (V: $400 \mathrm{~nm}$ ) transmitted together as different bit sequences shine on the front of the device.

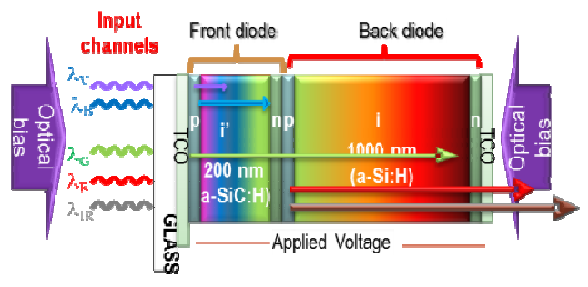

Fig. 1. Device configuration and operation 
The combined optical signal is analyzed by reading out the photocurrent, under either front or back optical bias provided by constant illumination with $(390 \mathrm{~nm})$ LEDs [3].

In Fig. 2 the optical gain $(\alpha)$, defined as the ratio between the spectral photocurrent with and without applied optical bias, is displayed under front irradiation. The background intensity $(\phi)$ was changed between $5 \mu \mathrm{Wcm}^{-2}$ and $3800 \mu \mathrm{Wcm}^{-2}$. Results show that the optical gains have opposite behaviour under front and back irradiations. Under front irradiation and low flux, the gain is high in the infrared region, presents a well-defined peak at $750 \mathrm{~nm}$ and strongly quenches in the visible range, as shown in Fig. 2a).
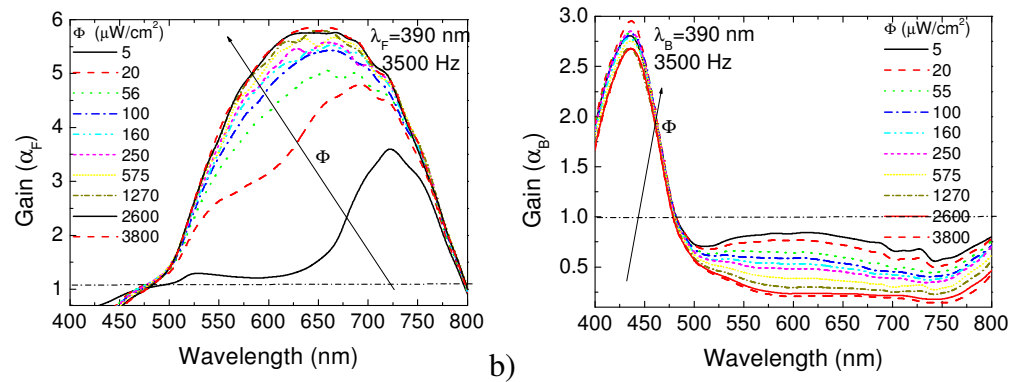

Fig. 2. Spectral gain $\left(\alpha_{B}\right)$ at $\lambda=390 \mathrm{~nm}$ under a) front and $b$ ) back irradiation

As the power intensity increases the peak shifts to the visible range and the spectral sensitivity can be deconvoluted into two peaks, one in the red range that slightly increases with the background intensity and another, in the green range, that strongly increases with it. In the blue range the gain is much lower. This shows the controlled long-pass filtering properties of the device. Depending on the background intensity selects the infrared or the visible spectral ranges; low fluxes select the near infrared region and cuts the visible one, the reddish part of the spectrum is selected at medium fluxes, and high fluxes tune the red/green ranges [5]. Fig. 2b) shows the photocurrent gain under back irradiation. The background intensity $(\phi)$ was changed between $5 \mu \mathrm{Wcm}^{-2}$ and $3800 \mu \mathrm{Wcm}^{-2}$. Results show that under back irradiation, the gain is high in the blue region, presents a well-defined peak at $425 \mathrm{~nm}$ and strongly quenches above $500 \mathrm{~nm}[6]$.

\section{Experimental Setup}

The optical bias as a steady lighting is used in different applications of this device namely as a WDM communication element and digital light logical functions.

To use the device as a volatile memory the optical bias paradigm is changed and looked upon as two light Control signals; the Front Control and the Back Control. The operation has three phases, the Control, the Hibernation and the Data phase. During the Control phase only the Front Control and the Back Control signals may be presented to the sensor. The Hibernation phase has no illumination signals; the sensor 
is in complete darkness. During the Data phase the sensor is subjected to the Data Sense signal.

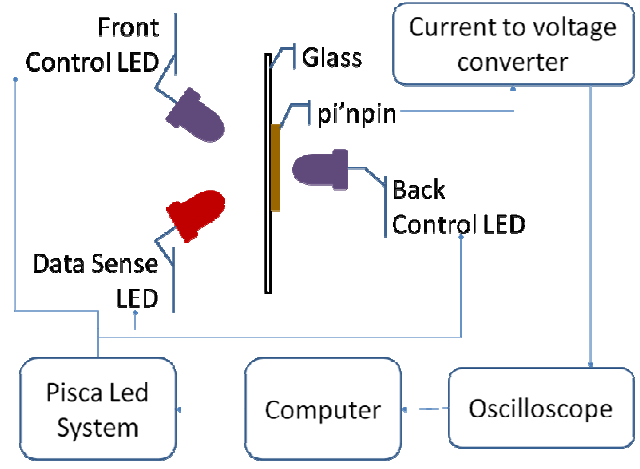

Fig. 3. LEDs, signals and the pi'npin device.

The Front and Back Control signals use a $390 \mathrm{~nm}$ LED, set with an intensity current of $5 \mathrm{~mA}$ and $20 \mathrm{~mA}$ respectively. These values differ, so that the output of the pi'npin may be similar. The Data Sense signal is a single $626 \mathrm{~nm}$ LED channel pulsed at $6000 \mathrm{H}_{\mathrm{z}}$, set at an intensity current of $5 \mathrm{~mA}$. These intensity current values were chosen so as to allow a clear visualization of the memory effect. The relative positioning of the LEDs and the pi'npin device can be visualized in Fig. 3.

A signal labeled as Dark is the photocurrent output when there are no Control signals and only the Data Sense signal is applied. It is used as a reference.

The double pi'npin sensor has a noise margin of 8nA [7].

\section{Memory Effects}

To introduce the memory theme a simple experiment is shown in Fig. 4a). On the top of the figure, there are the signals to guide the eyes.
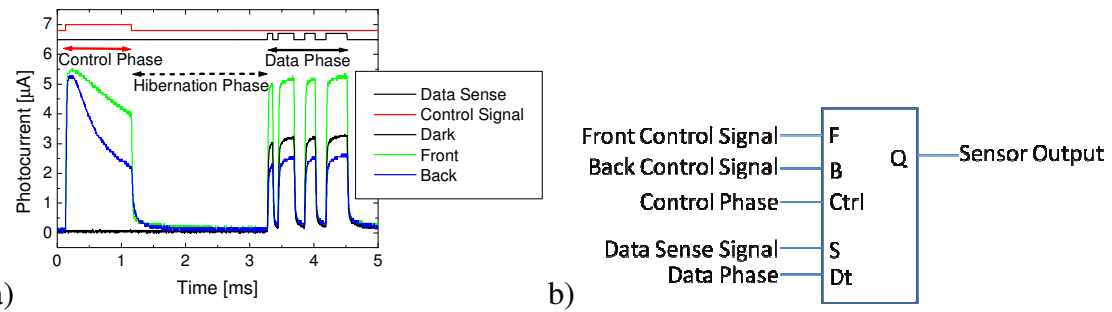

Fig. 4. a) Three outputs represented by the different conditions: Dark, Front Control and Back Control, on the same plot. b) Digital component of the equivalent pi'npin setup.

The experiment shown in Fig. 4a) is the result of the following sequential actions:

1- The Data Sense signal is applied and the pi'npin sensor's output is plotted and named Dark in Fig. 4a). 
2- The Back Control signal is applied during $1 \mathrm{~ms}$, followed by complete darkness during $2 \mathrm{~ms}$, and then the Data Sense signal is applied. The output is plotted and named Back in Fig. 4a).

3- The Front Control signal is applied during $1 \mathrm{~ms}$, followed by complete darkness during $2 \mathrm{~ms}$, and then the Data Sense signal is applied. The output is plotted and named Front in Fig. 4a).

These sequential actions were spaced in time between them by more than 2 minutes. This to ensure that one action does not have a big influence on the next one.

Observing Fig. 4 between 3 and $5 \mathrm{~ms}$ it is notable the reduced output of the Back in relation to the Dark, and the enhanced output of the Front in relation to the Dark. There is an influence of the Control signal over the Data Sense signal. Let the output be represented by the function $f$ (control, sense).

To model this effect a digital component is presented in Fig. 4b). The digital component has all the input signals on the left hand side and the output signal on the right hand side.

The Control Phase input enables the Front Control and the Back Control signal, and the Data Phase input enables the Data Sense signal.

The operation of the digital component is better described with the aid of Table 1.

Table 1. Automata state variable table

\begin{tabular}{|c|c|c|c|c|c|c|c|c|}
\hline \multirow{2}{*}{ Line } & \multicolumn{5}{|c|}{ Inputs } & \multicolumn{2}{c|}{ Internal State $_{|c|}$} & Output \\
\cline { 2 - 9 } & $\mathrm{F}$ & $\mathrm{B}$ & $\mathrm{Ctrl}$ & $\mathrm{S}$ & $\mathrm{Dt}$ & Store $_{\text {actual }}$ & Store $_{\text {next }}$ & Q \\
\hline 1 & $\mathrm{~F}$ & 0 & 1 & - & 0 & - & $\mathrm{F}$ & - \\
\hline 2 & 0 & $\mathrm{~B}$ & 1 & - & 0 & - & $\mathrm{B}$ & - \\
\hline 3 & - & - & 0 & - & 0 & Store $_{\text {actual }}$ & Store $_{\text {actual }}$ & 0 \\
\hline 4 & - & - & 0 & $\mathrm{~S}$ & 1 & Store $_{\text {actual }}$ & Store $_{\text {actual }}$ & f(Store $\left._{\text {actual }}, \mathrm{S}\right)$ \\
\hline 5 & - & - & 1 & - & 1 & - & - & - \\
\hline
\end{tabular}

Presented in Table 1 are the automata transition states of the digital component. In this table the (-) symbol represents a don't care value, which means that whatever the value that the variable has on that state, it does not interfere on the subsequent action. When used as an output it means that the output in that case should not be considered. The (1) symbol means that the signal is active, and the (0) symbol that the signal is not active. The internal state is defined by variable Store, which is represented as Store $_{\text {actual }}$ indicating the value that it holds in the present state. Store ${ }_{n e x t}$ represents the value that Store will hold due to the input values. Line 1 indicates that when the Control phase is active and the input Front Control as a value $F$ the next state will be set with that value. Line 2 indicates that when the Control phase is active and the input Back Control as a value B the next state will be set with that value. Line 1 and 2 also imply that the Front and Back Control are not set simultaneously. Line 3 shows that when there is neither a Control phase nor a Data phase the internal state variable will hold the same value; i.e. during the Hibernation phase the stores value does not change, and that the output is inactive. Line 4 indicates that when the Data phase is active the sensor output is a function of the present value of the internal state variable Store and of the Data Sense signal S; output $=f($ control, sense $)$. Line 5 indicates that 
if the Control phase and Data phase are set simultaneously the component will have unpredictable results.

The three phases, Control, Hibernation and Data have to be studied for the understanding of the memory effect. The approach used will focus on the duration in time of the three phases. Some time setting will be fixed and others vary to better observe and understand the results.

\subsection{Control Signal Phase Duration}

This study uses Control signals with different time duration, from 1 to $5 \mathrm{~ms}$. After each Control signal, follows a fixed Hibernation phase that lasts $3 \mathrm{~ms}$ after which a Data Sense signal is applied. Fig. 5a) presents the results of the applied Front Control signals, and Fig. 5b) shows the results for the applied Back Control signals.
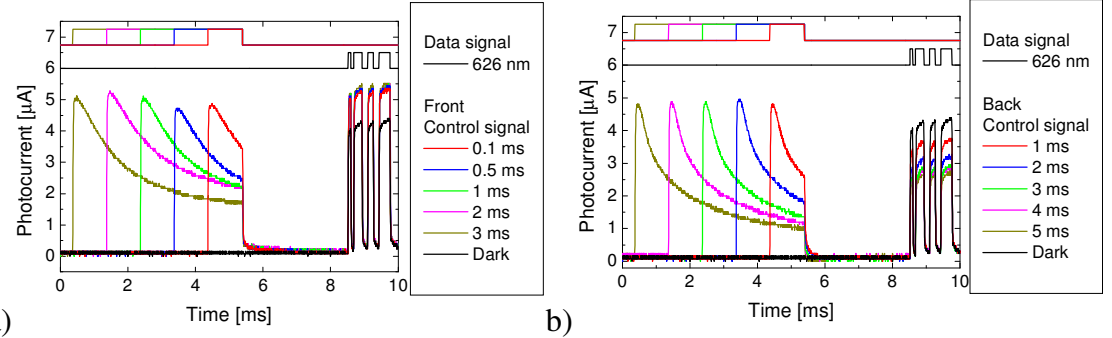

Fig. 5. a) Front Control signals and b) Back Control signals, with different lengths in time followed by a fixed Hibernation time and the same Data Sense signal.

Results show that there is almost no difference to the output produced by the applied Data Sense signal despite differences in the length of the applied Front Control signal. The Data Sense signal output is enhanced related to the Dark reference which is in conformance with the spectral gain presented in section 2. The results also show that higher duration of the Back Control signals reduces more the output of the applied signal when comparing it to the Dark reference. Comparing both Fig. 5 a) and b) on the Data Sense signal output, it is possible to infer the side on which a Control signal was set.

\subsection{Constant Control Plus Hibernation Time}

For visualization purposes the sum of the Control phase time plus the Hibernation phase time remains constant with a value of $3.1 \mathrm{~ms}$. On successive experiments the focus will be on the Control signal duration, from $0.1 \mathrm{~ms}$ to $3 \mathrm{~ms}$. The aim is to have the highest difference in photocurrent of the Data Sense signal output comparing the results of the applied Front Control or Back Control signal. The Data Sense signal is the same on all experiments, and is a random digital sequence.

Presented in Fig. 6a) is the experimental result of different Front Control signals followed by a short sequence of a Data Sense signal and in Fig. 6b) the experimental result of different Back Control signals followed by the same Data Sense signal. 

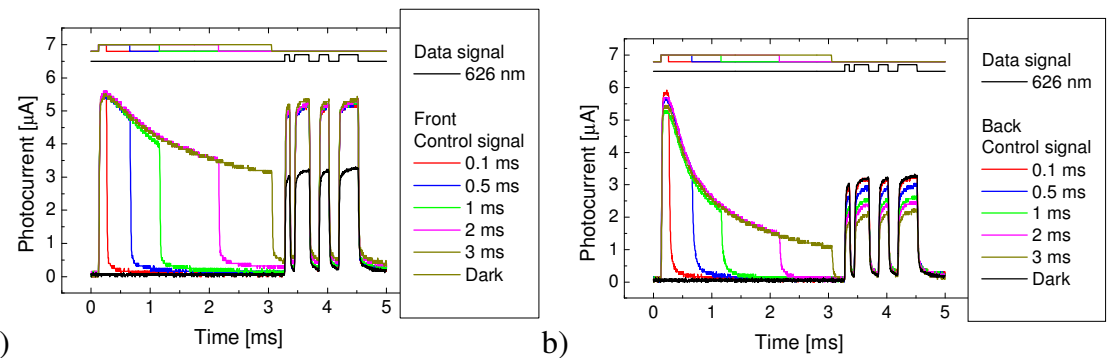

Fig. 6. a) Front Control signals and b) Back Control signals, with different lengths in time followed by the same Data Sense signal.

Results show that there is almost no difference to the output produced by the applied Data Sense signal despite differences in the length of the applied Front Control signal. The Data Sense signal output is enhanced related to the Dark reference which is in conformance with the spectral gain presented in section 2. Results also show that the output produced by the applied Data Sense signal reduces with the increase of the duration in time of the Back Control signal. The Data Sense signal output is quenched related to the Dark reference which is in conformance with the spectral gain presented in section 2. Observing both figures Fig. 6a) and b) it is clear that, the longer in time the Front and Back Control signals are the higher the photocurrent output difference between the Data Sense signals is.

\subsection{Control Signal Current Intensity}

The Control Signal is applied with different current intensities, $1 \mathrm{~mA}$ to $30 \mathrm{~mA}$, followed by a fixed Hibernation time of $3 \mathrm{~ms}$, and then a Data Sense signal is applied.

Presented in Fig. 7a) is the result of each Front Control signal applied to the pi'npin and Fig. 7b) shows the results for the applied Back Control signals.
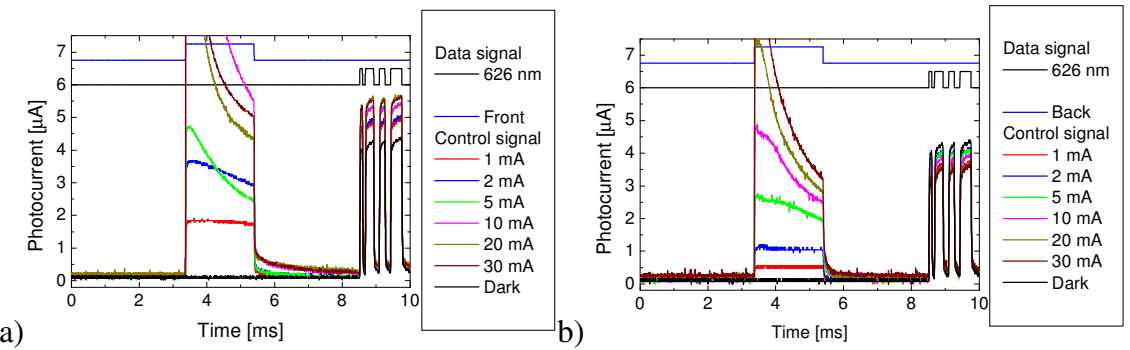

Fig. 7. a) Front Control and b) Back Control signals, with different current intensities followed by a fixed Hibernation time and the same Data Sense signal.

The figures are truncated for the higher currents because its representation does not concern the result discussion, and would otherwise prevent a better visualization of the results. The results show that the higher the Front Control signal current intensity is, the higher is the output signal, when comparing to the Dark reference. Results also 
show that the output of the Data Sense signal is slightly reduced, when compared to the Dark signal, for increasing current intensities of the Back Control signal.

Observing both figures Fig. 7 a) and b), and regarding the Dark reference, it is better to use the highest current possible for the Back and Front Control signals due to the fact that they provide Data Sense signal outputs with greater differences to the Dark reference.

\section{Conclusions}

By comparing the amplitudes of the Dark signal output to Data Sense signal output it is possible to know if the Control signal beforehand was a Control Front or a Control Back signal. The Control Front and Control Back signals are pulsed ultra-violet wavelengths. The Data Sense signal is a $626 \mathrm{~nm}$ wavelength signal. The memory stores which of the control signal was used last, either the Control Front signal or the Control Back signal. The memory is read by the Data Sense signal indicating if the Control Front signal was used or if the Control Back signal was used. A volatile memory can be built using a single pi'npin device.

Acknowledgments. This work was supported by FCT (CTS multi annual funding) through PIDDAC Program funds and PTDC/EEA-ELC/111854/2009 and PTDC/EEA-ELC/120539/2010.

\section{References}

1. R. Guo, L. You, Y. Zhou, Z. S. Lim, X. Zou, L. Chen, R. Ramesh, and J. Wang, "Nonvolatile memory based on the ferroelectric photovoltaic effect.," Nat. Commun., vol. 4, p. 1990, Jan. 2013.

2. G. de Ruiter, L. Motiei, J. Choudhury, N. Oded, and M. E. van der Boom, "Electrically Addressable Multistate Volatile Memory with Flip-Flop and Flip-Flap-Flop Logic Circuits on a Solid Support," Angew. Chemie, vol. 122, no. 28, pp. 4890-4893, May 2010.

3. M. A. Vieira, M. Vieira, J. Costa, P. Louro, M. Fernandes, and A. Fantoni, "Double Pin Photodiodes with Two Optical Gate Connections for Light Triggering.," Sensors \& Transducers, vol. 10, no. Special issue, pp. 96-120, 2011.

4. "Software system engineering research group (SSERG) - http://www.sserg.org/." https://tuhat.halvi.helsinki.fi/portal/files/28513674/cbse13_proceedings.pdf.

5. M. Vieira, M. A. Vieira, I. Rodrigues, V. Silva, and P. Louro, "Tuning optical a-SiC/a-Si active filters by UV bias light in the visible and infrared spectral ranges," Phys. status solidi, vol. 11, no. 11-12, pp. 1674-1677, Nov. 2014.

6. M. Vieira, M. A. Vieira, V. Silva, I. Rodrigues, and P. Louro, "Near-UV background as a bridge between visible and infrared communication," (In Publ.

7. V. Silva, M. A. Vieira, P. Louro, M. Barata, and M. Vieira, "Simple and complex logical functions in a $\mathrm{SiC}$ tandem device," in Technological Innovation for Collective Awareness Systems, 2014, pp. 592-601. 